\title{
Desactivación de la Fotoluminiscencia de un Complejo de Rutenio Anclado a Nanocristales de Dióxido de Titanio Empleando Cobaloximas
}

\author{
Sebastián G. Pizarro, Guillermo Cabrera, Guillermo A. Saá y Álvaro Delgadillo* \\ Departamento de Química, Facultad de Ciencias, Universidad de La Serena, Casilla 599, Benavente 980; \\ La Serena, Chile (e-mail: adelgadillo@userena.cl)
}

${ }^{*}$ Autor a quien debe ser dirigida la correspondencia

Recibido Feb. 7, 2014; Aceptado Abr. 3, 2014; Versión final recibida May. 9, 2014

\begin{abstract}
Resumen
La desactivación del estado excitado del complejo $\left[\mathrm{Ru}(\mathrm{deeb})(\mathrm{bpy})_{2}\right]\left(\mathrm{PF}_{6}\right)_{2}$ (donde bpy es 2,2'-bipiridina y deeb es 4,4'-(COOEt) 22,2'-bipiridina) fue estudiada en disolución de acetonitrilo y también anclado sobre una película delgada de nanocristales de dióxido de titanio. Para desactivar el estado excitado del compuesto de rutenio se emplearon los complejos [Co $\left.(\mathrm{py})(\mathrm{Cl})(\mathrm{dmgH})_{2}\right]$ y $\left[\mathrm{Co}(\mathrm{py})(\mathrm{Bu})(\mathrm{dmgH})_{2}\right]$ (donde py es piridina, $\mathrm{Bu}$ es $\mathrm{n}$-butilo y dmgH es dimetilglioxamato). Estas cobaloximas pueden desactivar de manera eficiente el estado excitado tanto en disolución como soportado sobre dióxido de titanio. El proceso de desactivación puede ser descrito mediante el modelo Stern-Volmer, cuyo análisis indica que el proceso de desactivación está dominado por procesos de difusión y es coherente con un proceso de transferencia de electrones foto inducido.
\end{abstract}

\section{Photoluminiscence Quenching of Ruthenium Complexes Anchored to Nanocrystalline Titanium Dioxide using Cobaloximes}

\begin{abstract}
The quenching of the excited state of $\left[\mathrm{Ru}(\mathrm{deeb})(\mathrm{bpy})_{2}\right]\left(\mathrm{PF}_{6}\right)_{2}$ where bpy is $2,2^{\prime}$-bipyridine and deeb is $4,4^{\prime}$ (COOEt)2-2,2'-bipyridine was studied in fluid acetonitrile solution and anchored to nanocrystalline titanium dioxide thin films. To quench the excited state, the cobaloximes $\left[\mathrm{Co}(\mathrm{py})(\mathrm{Cl})(\mathrm{dmgH})_{2}\right]$ and $\left[\mathrm{Co}(\mathrm{py})(\mathrm{Bu})(\mathrm{dmgH})_{2}\right] \quad$ where py is pyridine, $\mathrm{Bu}$ is $\mathrm{n}$-butyl and $\mathrm{dmgH}$ is dimethylglyoximate were used. These cobaloximes are efficient quenchers of the excited state both in fluid solution and when the ruthenium complex is bound to titanium dioxide. The quenching process can be adequately described using the SternVolmer model, which indicates that quenching is dominated by diffusion processes and is coherent with a photo induced electron transfer process.
\end{abstract}

Keywords: ruthenium, cobaloximes, titanium dioxide, excited state quenching, Stern-Volmer model 


\section{INTRODUCCIÓN}

Desde que los complejos de cobalto con ligando del tipo dioxima fueron propuestos como modelos de la vitamina B12 (Schrauzer, 1968) han concitado un gran interés debido principalmente a que también pueden actuar como catalizadores, tanto en la deshalogenación reductiva de compuestos órgano-halogenados persistentes (McCauley et al., 2002) como en la generación electro-catalítica de di hidrogeno con bajos sobre potenciales en presencia de una fuente de protones (Dempsey et al., 2009; Du et al., 2009; Zhang et al., 2010; Probst et al., 2011).

La característica fundamental que presentan estos compuestos, y que los lleva a ser utilizados en estas aplicaciones, es la capacidad de estabilizar a los centros metálicos de Co en los estados de oxidación formales III, II y I. Los complejos de $\mathrm{Co}$ (I) son conocidos como súper nucleófilos y la mayoría de las aplicaciones catalíticas se asocian a este estado de oxidación (Schrauzer, 1968).

Por lo general los compuestos de Co(I) son preparados a partir de complejos de Co(III) mediante la adición de agentes reductores en fase homogénea (McCauley et al., 2002) o mediante procesos fotoquímicos (Dong et al., 2011). Una de las desventajas que presentan los procesos catalíticos de cobalto en fase homogénea radica en la dificultad de separar o aislar los componentes de interés luego de que la reacción ha sido completada. Por esta razón, nuestro grupo de investigación está interesado en determinar las condiciones necesarias para generar complejos de $\mathrm{Co}(\mathrm{I})$ por medio de reacciones de transferencia de electrones fotoinducida en un sistema heterogéneo.

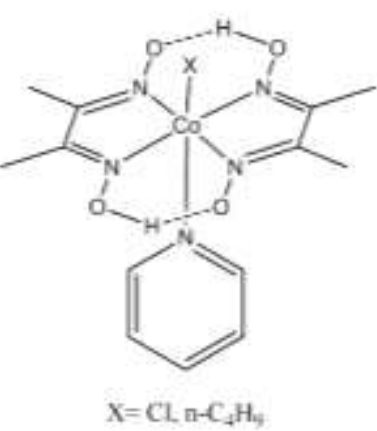

(a)

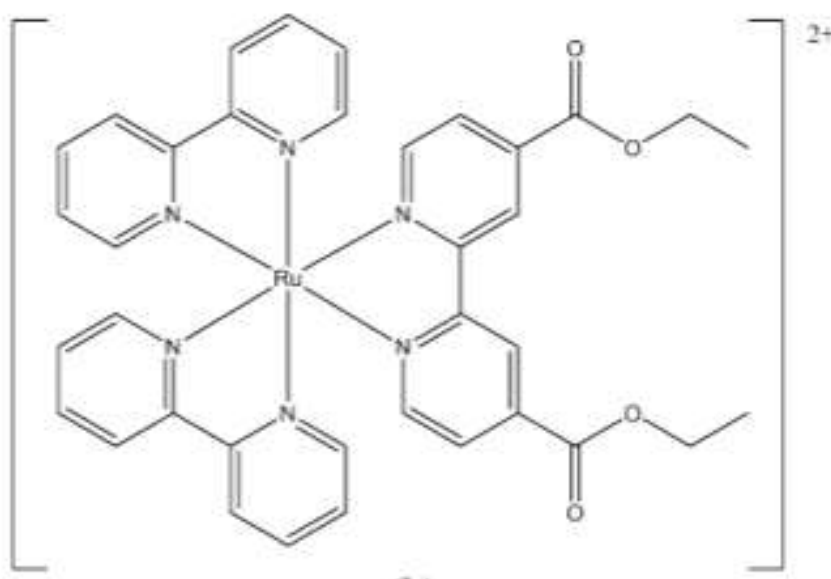

(b)

Fig. 1: Complejos empleados en este estudio: (a) $\left[\mathrm{Co}(\mathrm{py})(\mathrm{Cl})(\mathrm{dmgH})_{2}\right]$ y (b) $\left[\mathrm{Ru}(\mathrm{deeb})(\mathrm{bpy})_{2}\right]\left(\mathrm{PF}_{6}\right)_{2}$.

Los complejos polipiridínicos de rutenio han sido ampliamente estudiados por sus propiedades fotofísicas. Presentan bandas de absorción de transferencia de carga metal ligando, TCML, en la región visible del espectro electromagnético por lo que son fuertemente coloreados. La irradiación con luz de energía apropiada en estas bandas de absorción generan estados excitados que son mejores oxidantes y reductores que el estado basal (Kalyanasundaram, 1992). Una manera sencilla de estudiar los estados excitados generados es monitorear su fotoluminiscencia en estado estacionario, ya que sus variaciones pueden ser asociadas a procesos de transferencia de electrones o energía (Meyer, 1982).

Por otro lado, varios óxidos metálicos pueden ser preparados empleando métodos solvotermales los que permiten controlar parámetros como su fase y tamaño (Galeano et al., 2013; Heimer et al., 1996). Los compuestos de rutenio, que presenten grupos funcionales adecuados, pueden ser soportados sobre superficies de óxidos metálicos sin cambiar sustancialmente sus propiedades fotofísicas, conformando de esta manera sistemas supramoleculares heterogéneos, que pueden ser empleados en la reducción de especies en disolución.

En este trabajo se presenta el estudio de la desactivación del estado excitado de un complejo de rutenio inducido por una cobaloxima, Fig. 1. El complejo de rutenio en estudio es el $\left[\mathrm{Ru}(\mathrm{deeb})(\mathrm{bpy})_{2}\right]\left(\mathrm{PF}_{6}\right)_{2}$ (donde bpy es 2,2'-bipiridina y deeb es el 4,4'-(COOEt)2-2,2'-bipiridina). La desactivación se llevó a cabo en disolución de acetonitrilo y también adsorbido sobre películas delgadas de nanocristales de dióxido de titanio, con el propósito de contrastar un sistema homogéneo frente a uno heterogéneo. Para inducir la desactivación del estado excitado se emplearon las siguientes cobaloximas: $\left[\mathrm{Co}(\mathrm{py})(\mathrm{Cl})(\mathrm{dmgH})_{2}\right]$ y $\left[\mathrm{Co}(\mathrm{py})(\mathrm{Bu})(\mathrm{dmgH})_{2}\right]$ donde py es piridina, Bu es n-butilo y dmgH es dimetilglioximato. 


\section{MATERIALES Y MÉTODOS}

\section{Reactivos}

Todos los reactivos y solventes fueron utilizados sin previa purificación: Acetonitrilo (99,9\%, Merck), Isopropóxido de titanio(IV) (97\%, sigma-aldrich), Cloruro de cobalto(II) hexahidratado (98\%, Fluka), Cloruro de rutenio(III) hidrato (99\%, precious metals online), 1-yodobutano (99\%, Merck), dimetilglioxima (97\%, Fluka), 4,4'-dimetil-2,2'-dipiridil (99\%, sigma-aldrich). Los compuestos de coordinación $\left[\mathrm{Ru}(\mathrm{deeb})(\mathrm{bpy})_{2}\right]\left(\mathrm{PF}_{6}\right)_{2}$ (Meyer, 1999), [Co(py) $\left.(\mathrm{Cl})(\mathrm{dmgH})_{2}\right]$ y [Co(py) $\left.(\mathrm{Bu})(\mathrm{dmgH})_{2}\right]$ (Dutta et al., 2009) fueron preparados de acuerdo a los procedimientos reportados en literatura.

El dióxido de titanio mesoporoso fue preparado por técnicas sol-gel de acuerdo a lo reportado en literatura (Heimer et al., 1996). Brevemente, dióxido de titanio es generado por la hidrólisis ácida de isopropóxido de titanio(IV), la dispersión obtenida es peptizada en condiciones solvotermales a $120 \stackrel{\circ}{\mathrm{C}}$ por 8 horas.

Para las determinaciones espectrofotométricas las partículas de $\mathrm{TiO}_{2}$ se depositaron en vidrios portaobjetos de microscopio $(50 \times 12,5 \times 1 \mathrm{~mm})$ de manera que podían ser introducidas en forma diagonal en una cubeta de cuarzo que contiene como solvente acetonitrilo. Para mediciones electroquímicas se deposito el dióxido de titanio sobre el lado conductor de vidrios con un recubrimiento de oxido de estaño dopado con flúor (Pilkington glass). Antes de realizar la adsorción de los compuestos de interés el $\mathrm{TiO}_{2}$ fue tratado colocando las películas en una disolución acuosa a pH 11 durante 1 hora y posteriormente fue lavado utilizando acetonitrilo para así modificar la posición del filo de la banda de conducción impidiendo así la inyección de electrones del estado excitado de rutenio al dióxido de titanio (Hagfeldt y Graetzel, 1995).

Las películas delgadas de $\mathrm{TiO}_{2}$ se sumergieron en disoluciones de concentración micromolar del complejo $\left[\mathrm{Ru}(\mathrm{deeb})(\mathrm{bpy})_{2}\right]\left(\mathrm{PF}_{6}\right)_{2}$ en acetonitrilo por un periodo de 24 horas. El recubrimiento de superficie, $\Gamma$ (mol $\mathrm{cm}^{-2}$ ), fue determinado espectroscópicamente de acuerdo a la siguiente ecuación.

$A=\varepsilon \times \Gamma \times 1000$

Donde $A$ es la absorbancia de la película sensibilizada de dióxido de titanio medida en modo transmisión y $\varepsilon\left(\mathrm{M}^{-1} \mathrm{~cm}^{-1}\right)$ es el coeficiente de absorción registrado en disolución (Galoppini, 2002).

El potencial de reducción del estado excitado, $E_{1 / 2}\left(R^{11 / / I^{*}}\right)$, fue calculado a partir de los potenciales de reducción de estado basal y de la energía libre almacenada en el estado excitado TCML térmicamente equilibrado, $\Delta \mathrm{Gee}_{\mathrm{e}}$

$\mathrm{E}_{1 / 2}\left(\mathrm{Ru}^{\mathrm{III} / \mathrm{II} *}\right)=\mathrm{E}_{1 / 2}\left(\mathrm{Ru}^{\mathrm{III} / \mathrm{II}}\right)-\Delta \mathrm{G}_{\mathrm{ee}}$

$\Delta G_{e e}$ se estimó trazando una línea tangente al lado de mayor energía del espectro de fotoluminiscencia (Meyer).

\section{Mediciones físicas}

El espectro de absorción fue medido en un espectrofotómetro Spekol UV-Vis 1200, los experimentos de fotoluminiscencia en estado estacionario se realizaron a temperatura ambiente en un equipo Shimadzu RF$5301 \mathrm{PC}$, la longitud de onda de excitación fue $450 \mathrm{~nm}$. Las medidas electroquímicas se llevaron a cabo en acetonitrilo 0,1 M TBAPF $_{6}$ utilizando un potenciostato Princeton Applied Research PG 580 con la configuración clásica de tres electrodos consistentes en un electrodo de disco plano de Pt, un alambre de electrodo auxiliar de Pt y un electrodo de referencia de $\mathrm{Ag} / \mathrm{AgCl}$. Para la determinación del potencial de reducción del centro de rutenio anclado a dióxido de titanio se empleo como electrodo de trabajo una película de dióxido de titanio soportada en un vidrio conductor FTO, el electrolíto empleado para ese experimento fue perclorato de litio $0.1 \mathrm{M}$.

\section{RESULTADOS Y DISCUSIONES}

La Fig. 2 muestra espectro de absorción y fotoluminiscencia del complejo $[R u(d e e b)(b p y) 2]^{2+}$ en disolución y también anclado sobre una película de $\mathrm{TiO}_{2}$. En ambos casos se utilizó como disolvente acetonitrilo saturado con argón para eliminar la presencia del oxígeno. Los datos fotofísicos y electroquímicos de este compuesto están resumidos en la tabla 1. En la Tabla, (a) todas las mediciones fueron realizadas a temperatura ambiente. Las películas delgadas de $\mathrm{TiO}_{2}$ se sumergieron en acetonitrilo; (b) Datos tomados de la literatura (Bergeron y Meyer, 2003) 


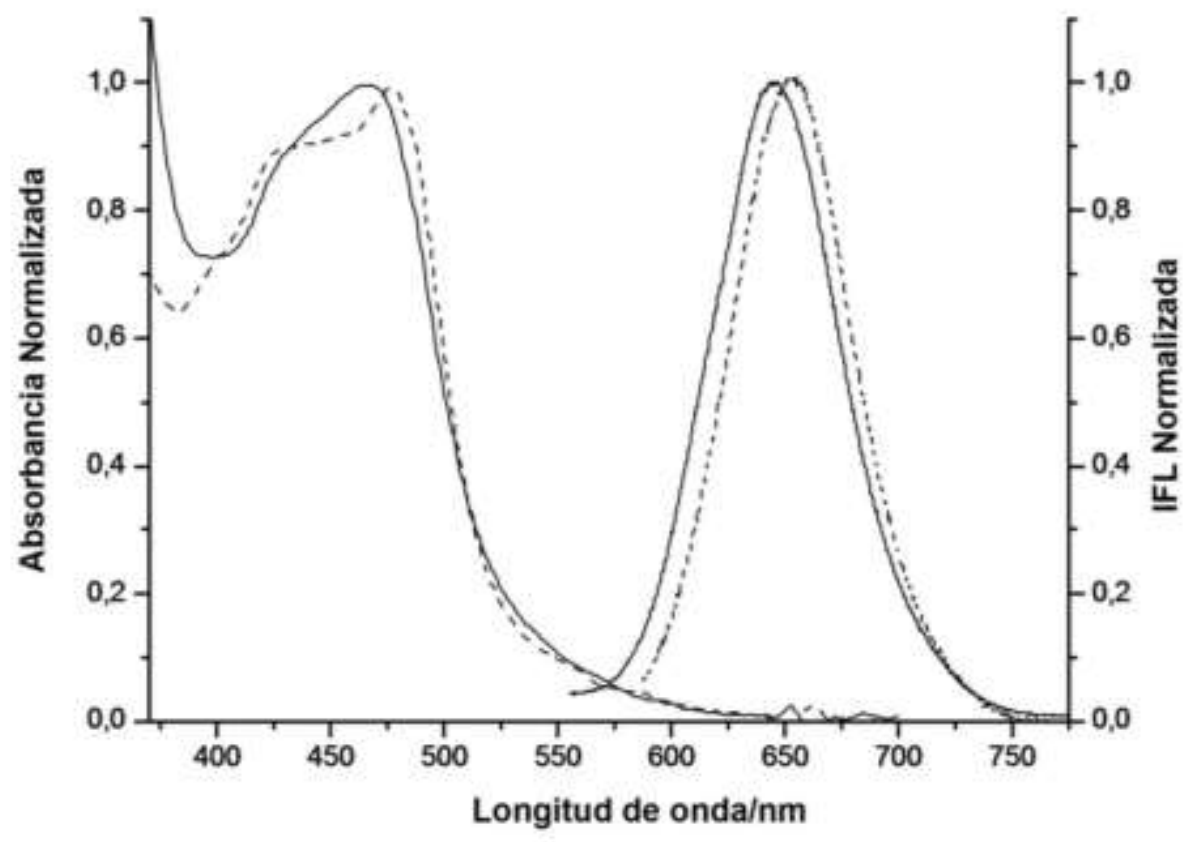

Fig. 2. Absorbancia y fotoluminiscencia normalizada en estado basal de $\left[R u(d e e b)(b p y)_{2}\right]\left(P F_{6}\right)_{2}$ en acetonitrilo (línea discontinua), sobre $\mathrm{TiO}_{2}$ (línea continua).

Cuando el complejo $\left[\mathrm{Ru}(\mathrm{deeb})(\mathrm{bpy})_{2}\right]^{2+}$ es adsorbido sobre la superficie del óxido metálico semiconductor $\mathrm{TiO}_{2}$, se observa que presenta propiedades espectroscópicas similares a las que muestra en disolución líquida. La longitud de onda máxima, asociada a la banda de transferencia de carga metal ligando, TCML, está ligeramente desplazada a mayor energía presumiblemente debido a la unión del complejo con la superficie del óxido metálico.

A partir de los datos espectroscópicos del complejo $\left[\mathrm{Ru}(\mathrm{deeb})(\mathrm{bpy})_{2}\right]^{2+}$ se obtuvo un valor de recubrimiento de superficie de $2,3 \times 10^{-8} \mathrm{~mol} \mathrm{~cm}^{-2} \pm 0,3 \times 10^{-8} \mathrm{~mol} \mathrm{~cm}^{-2}$. La irradiación en la banda de TCML genera un estado excitado fotoluminiscente característico de los compuestos polipiridínicos de rutenio (Kalyanasundaram, 1992). La fotoluminiscencia se aprecia tanto en disolución líquida como también adsorbido sobre la superficie del $\mathrm{TiO}_{2}$, observándose también un leve desplazamiento de la longitud de onda máxima hacia zonas de mayor energía.

Para poder registrar los espectros de fotoluminiscencia del complejo adsorbido sobre $\mathrm{TiO}_{2}$, la superficie del óxido metálico fue tratada con una disolución básica, debido a que en otras condiciones la fotoluminiscencia disminuye de manera significativa por la inyección interfacial de electrones a la banda de conducción del dióxido de titanio (Kelly et al., 1999).

Tabla 1: Propiedades fotofísicas y potenciales de reducción de $\left[\mathrm{Ru}(\mathrm{deeb})(\mathrm{bpy})_{2}\right]^{2+}$ sobre $\mathrm{TiO}_{2}$ y acetonitriloa

\begin{tabular}{|c|c|c|c|c|c|}
\hline & $\lambda_{\text {abs }}(\mathrm{nm})$ & $\lambda_{\mathrm{em}}(\mathrm{nm})$ & $\tau(\mu \mathrm{s})^{b}$ & E으 $\mathrm{Ru}(\mathrm{III} / \mathrm{II})$ & Eo $R u\left(I I I / I^{\star}\right)$ \\
\hline$\left[\mathrm{Ru}(\mathrm{deeb})(\mathrm{bpy})_{2}\right]^{2+}(\mathrm{sol})$ & $477 \pm 1$ & $652 \pm 5$ & 0.99 & $1.39 \pm 0.02$ & $-0.70 \pm 0.01$ \\
\hline$\left[\mathrm{Ru}(\mathrm{deeb})(\mathrm{bpy})_{2}\right]^{2+}\left(\mathrm{TiO}_{2}\right)$ & $465 \pm 1$ & $644 \pm 5$ & 1.04 & $1.39 \pm 0.02$ & $-0.74 \pm 0.01$ \\
\hline
\end{tabular}

El potencial de reducción del par redox $R u(I I I / I)$ fue registrado utilizando mediciones de voltametría cíclica. Estos potenciales en conjunto con los espectros de emisión permiten calcular el potencial de reducción del compuesto de rutenio en el estado excitado, los cuales se encuentran tabulados en la Tabla 1. Se puede apreciar que el potencial asociado al par redox $\mathrm{Ru}\left(\mathrm{III} / \mathrm{II}^{*}\right)$ es levemente más negativo cuando el complejo de rutenio esta adsorbido sobre dióxido de titanio.

A medida que se adicionan las cobaloximas a las disoluciones en estudio se observa una disminución en la intensidad de la fotoluminiscencia asociada a la desactivación del estado excitado del complejo de rutenio, en las Fig. 3 y 4 se muestran los cambios en el espectro de emisión del complejo de rutenio tanto en disolución como adsorbido sobre dióxido de titanio. 


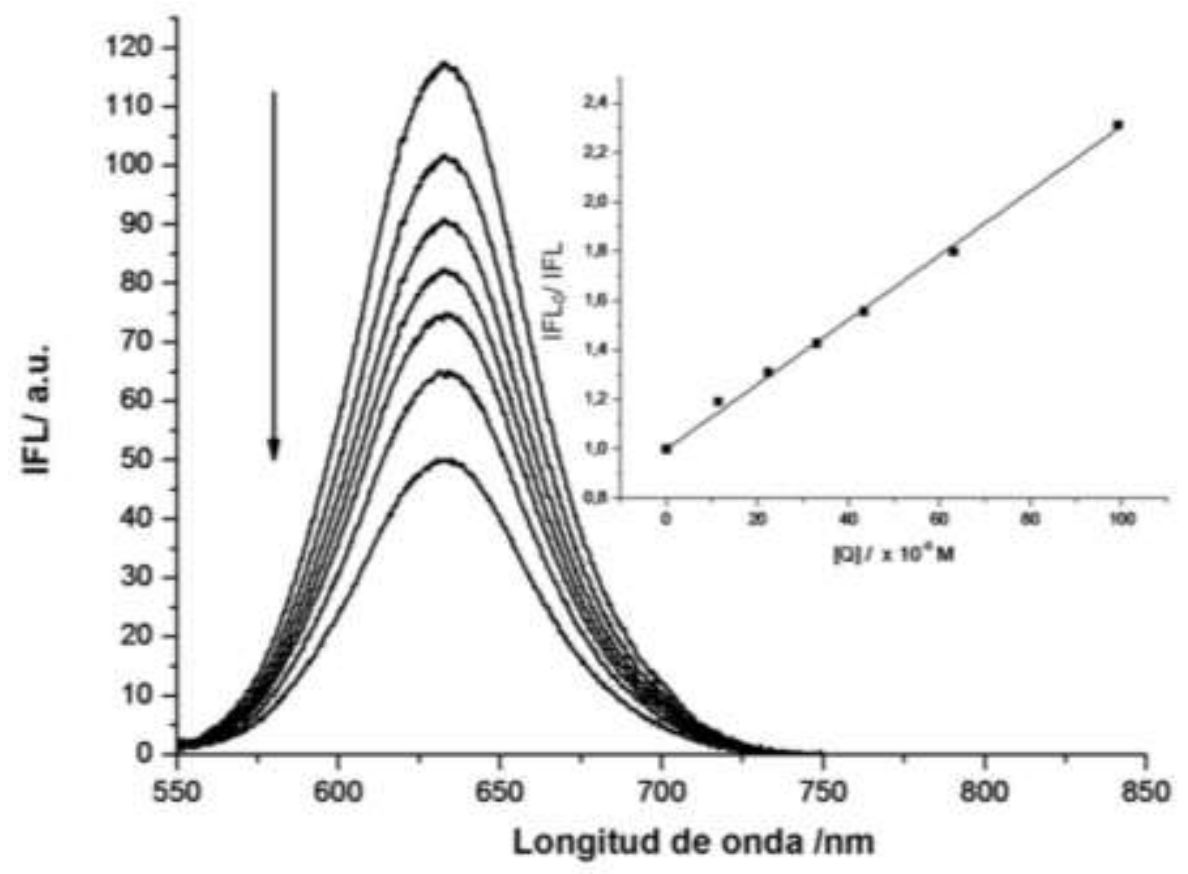

Fig. 3: Cambios en los espectros de emisión de $\left[R u(d e e b)(b p y)_{2}\right]^{2+}$ después de la adición de Cloro(piridina)bis(dimetilglioxamato)cobalto (III) en solución de acetonitrilo saturada en argón. El recuadro muestra el análisis grafico de Stern-Volmer

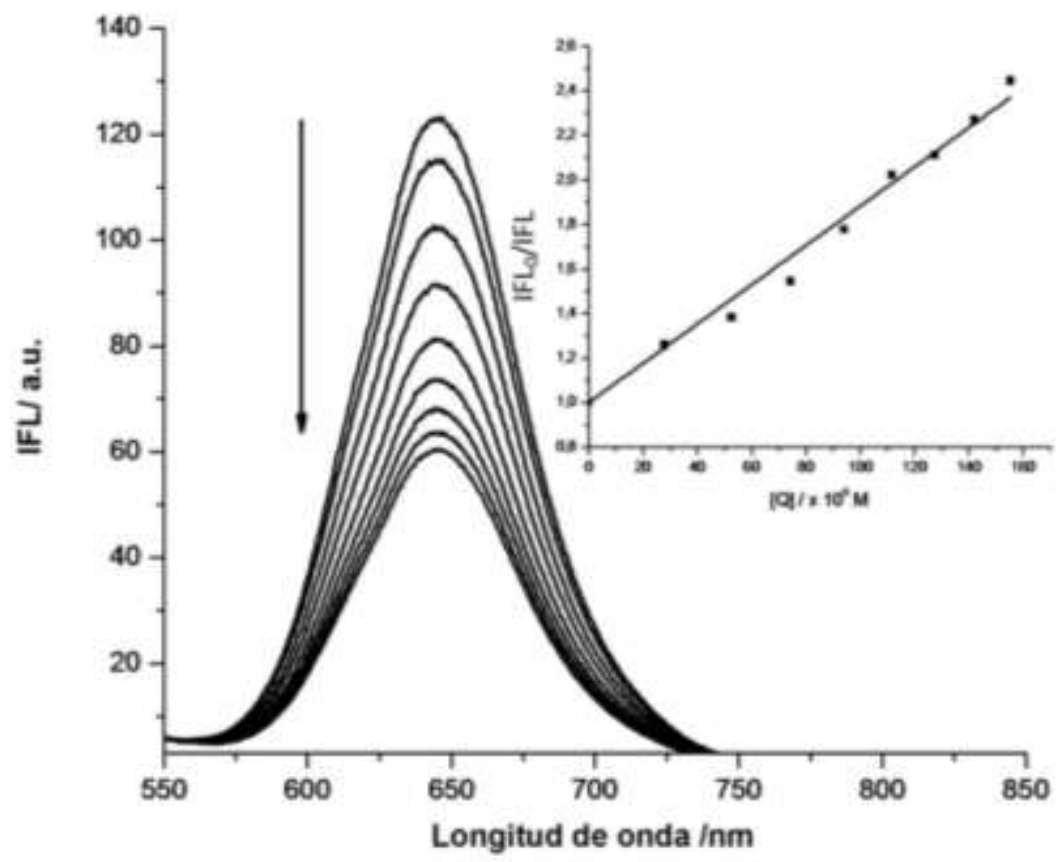

Fig. 4: Cambios en los espectros de emisión de $\left[\mathrm{Ru}(\mathrm{deeb})(\mathrm{bpy})_{2}\right]^{2+}$ adsorbido sobre $\mathrm{TiO}_{2}$ nanocristalino después de la adición de Cloro(piridina)bis(dimetilglioxamato)cobalto (III) en solución de acetonitrilo saturada en argón. El recuadro muestra el análisis grafico de Stern-Volmer.

La desactivación del estado excitado del complejo de rutenio inducido por la adición de cobaloxima puede ser analizado utilizando el modelo Stern-Volmer (3). Este modelo implica una competición entre el decaimiento inherente del estado excitado del complejo de rutenio y una desactivación bimolecular realizada por las cobaloximas. Los insertos en la figura 3 y 4 muestran el análisis grafico de Stern-Volmer.

$\frac{\mathrm{PLI}_{0}}{\mathrm{PLI}}=1+\mathrm{K}_{\mathrm{sv}}[\mathrm{Q}]$ 
En la ecuación 3 [Q] es la concentración del desactivador del estado excitado y $\mathrm{K}_{\mathrm{sv}}$ es la constante de SternVolmer, la que está relacionada con la constante de velocidad de desactivación, $\mathrm{k}_{\mathrm{q}}$, y el tiempo de vida del estado excitado, $\tau$, en ausencia de cobaloxima, (4).

$\mathrm{K}_{\mathrm{sv}}=\mathrm{k}_{\mathrm{q}} \times \tau$

Las contantes de Stern-Volmer y las constantes de velocidad de desactivación estimadas para los sistemas en estudio se resumen en la Tabla 2. Al comparar los valores de las constantes de los dos sistemas en estudio tanto en disolución líquida como adsorbido sobre el $\mathrm{TiO}_{2}$, se puede apreciar que el valor de las constantes encontradas para los sistemas en fase homogénea es mayor que cuando el compuesto de rutenio está anclado sobre la superficie de $\mathrm{TiO}_{2}$. Esta observación y la elevada magnitud de estas constantes sugieren que los procesos de desactivación del estado excitado están dominados por difusión. (Meyer, 1982).

Tabla 2: Constantes de velocidad de transferencia de electrones del estado excitado.

\begin{tabular}{lcccc}
\hline & \multicolumn{2}{c}{$\left[\mathrm{Co}(\mathrm{py})(\mathrm{Cl})(\mathrm{dmgH})_{2}\right]$} & \multicolumn{2}{c}{$\left[\mathrm{Co}(\mathrm{py})(\mathrm{Bu})(\mathrm{dmgH})_{2}\right]$} \\
\cline { 2 - 5 } & $\mathrm{K}_{\mathrm{Sv}}\left(\mathrm{M}^{-1}\right)$ & $\mathrm{k}_{\mathrm{q}} \times 10^{9}\left(\mathrm{M}^{-1} \mathrm{~s}^{-1}\right)$ & $\mathrm{Ksv}_{\mathrm{s}}\left(\mathrm{M}^{-1}\right)$ & $\mathrm{k}_{\mathrm{q}} \times 10^{9}\left(\mathrm{M}^{-1} \mathrm{~s}^{-1}\right)$ \\
\hline$\left[\mathrm{Ru}(\mathrm{deeb})(\mathrm{bpy})_{2}\right]^{2+}(\mathrm{sol})$ & $13000 \pm 200$ & $13,1 \pm 0,1$ & $10100 \pm 200$ & $10,2 \pm 0,1$ \\
{$\left[\mathrm{Ru}(\mathrm{deeb})(\mathrm{bpy})_{2}\right]^{2+}\left(\mathrm{TiO}_{2}\right)$} & $6500 \pm 300$ & $6,0 \pm 0,1$ & $3400 \pm 200$ & $3,3 \pm 0,1$ \\
\hline
\end{tabular}

La desactivación del estado excitado del complejo de rutenio podría proceder a través de un proceso de transferencia de energía, o mediante una transferencia de electrones fotoinducida. La transferencia de energía se puede descartar sobre la base del inexistente solapamiento espectral entre el donador, el estado excitado del compuesto de rutenio, y el aceptor, el estado basal de las cobaloximas ya que ninguna de las cobaloximas presenta absorción de radiación electromagnética a longitudes de onda mayores que $500 \mathrm{~nm}$ (Wen et al., 2011).

Esto sugiere que la desactivación del estado excitado del complejo $\left[\mathrm{Ru}(\mathrm{deeb})(\mathrm{bpy})_{2}\right]^{2+}$ podría proceder a través de un proceso de transferencia de electrones fotoinducido. Sin embargo, la fotogeneración de Co(I) es termodinámicamente inviable de acuerdo a la ecuación de Weller (Astruc, 2000). Experimentos de voltametria cíclica realizados en acetonitrilo indican que los potenciales de reducción del par Co(II/I) para las cobaloximas utilizadas son muy negativos, tal como se muestra en la tabla 3.

Tabla 3: Propiedades fotofísicas y potenciales de reducción de $\left[\mathrm{Co}(\mathrm{py})(\mathrm{Cl})(\mathrm{dmgH})_{2}\right]$ y $\left[\mathrm{Co}(\mathrm{py})(\mathrm{Bu})(\mathrm{dmgH})_{2}\right]$.

\begin{tabular}{lcccc}
\hline & $\lambda_{\text {abs }}(\mathrm{nm})$ & $\mathrm{E}_{1 / 2}$ Co $(\mathrm{II} / \mathrm{I})$ & $\mathrm{E}_{\mathrm{pc}}$ Co $(\mathrm{III} / \mathrm{II})$ & $\mathrm{E}_{\mathrm{pa}}$ Co $(\mathrm{II} / \mathrm{III})$ \\
\cline { 2 - 5 }$\left[\mathrm{Co}(\mathrm{py})(\mathrm{Cl})(\mathrm{dmgH})_{2}\right]$ & $400 \pm 1$ & $-1.03 \pm 0.02$ & $-0.63 \pm 0.01$ & $0.06 \pm 0.01$ \\
{$\left[\mathrm{Co}(\mathrm{py})(\mathrm{Bu})(\mathrm{dmgH})_{2}\right]$} & $425 \pm 1$ & $-1.67 \pm 0.02$ & - & - \\
\hline
\end{tabular}

Por lo tanto es más factible que la desactivación del estado excitado ocurra por la reducción de Co(III) a Co(II). Se conoce que las especies de Co(II) pueden sufrir reacciones de desproporción (Borgo, 2003), lo que permitiría generar $\mathrm{Co}(\mathrm{I})$ indirectamente. Con el fin de lograr la fotogeneración de $\mathrm{Co}(\mathrm{I})$, el potencial de reducción asociado al par $\mathrm{Co}$ (II/I) debe ser desplazado a valores más positivos, mientras que el potencial de reducción de estado excitado del complejo de rutenio debe ser desplazado a valores más negativos. Para lograr este objetivo se están realizando en nuestros laboratorios modificaciones estructurales a estos complejos

\section{CONCLUSIONES}

Las cobaloximas $\left[\mathrm{Co}(\mathrm{py})(\mathrm{Cl})(\mathrm{dmgH})_{2}\right]$ y $\left[\mathrm{Co}(\mathrm{py})(\mathrm{Bu})(\mathrm{dmgH})_{2}\right]$ desactivan de manera eficiente el estado excitado de $\left[\mathrm{Ru}(\mathrm{deeb})(\mathrm{bpy})_{2}\right]^{2+}$ tanto en disolución líquida como anclado sobre películas delgadas de dióxido de titanio nanocristalino. El proceso de desactivación observado se acerca al límite difusional y es coherente con un proceso de transferencia de electrones fotoinducido.

\section{AGRADECIMIENTOS}

Los autores agradecen el apoyo financiero de FONDECYT 1110648 y NUCLEUS MILLENNIUM No. P07006-F. 


\section{REFERENCIAS}

Astruc, D., Organometallic Chemistry and Catalysis, pp 83, Springer, Grenoble (2000).

Bergeron, B. V. y G. J. Meyer, Reductive Electron Transfer Quenching of MLCT Excited States Bound To Nanostructured Metal Oxide Thin Films, The Journal Of Physical Chemistry B, 107, 245-254 (2003).

Borgo, C. A., A. Lazarin, C. Davanzo, Y. Gushikem, Preparation and characterization of the cobaloxime complex and its use in fabrication of a modified electrode. An electrochemical experiment for undergraduate course, Quimica Nova, 26(6), 943-947 (2003).

Dempsey, J. L., B. S. Brunschwig, J. R. Winkler y H. B. Gray, Hydrogen Evolution Catalyzed by Cobaloximes, Accounts of Chemical Research, 42, 1995-2004 (2009).

Dong, J., M. Wang, P. Zhang, S. Yang, J. Liu, X. Li, y L. Sun, Promoting Effect of Electrostatic Interaction between a Cobalt Catalyst and a Xanthene Dye on Visible-Light-Driven Electron Transfer and Hydrogen Production, The Journal of Physical Chemistry C, 115, 15089-15096 (2011).

Du, P., J. Schneider, G. Luo, W. W. Brennessel, y R. Eisenberg, Visible Light-Driven Hydrogen Production from Aqueous Protons Catalyzed by Molecular Cobaloxime Catalysts, Inorganic Chemistry, 48, 4952-62 (2009).

Dutta, G., K. Kumar y B. D. Gupta, Cobaloximes with Bis(thiophenyl)glyoxime: Synthesis and Structure Property Relationship Study, Organometallics, 28, 3485-3491 (2009).

Galeano, L., J. A. Navío, G. M. Restrepo y J. M. Marín, Preparación de Sistemas Óxido de Titanio/Óxido de Silicio (TiO2/SiO2) mediante el Método Solvotérmico para Aplicaciones en Fotocatálisis, Información Tecnológica, 24(5), 81-92 (2013).

Galoppini, E., W. Guo, W. Zhang, P. G. Hoertz, P. Qu, G. J. Meyer, Long-Range Electron Transfer across Molecule-Nanocrystalline Semiconductor Interfaces Using Tripodal Sensitizers, Journal of the American Chemical Society, 124, 7801-7811 (2002).

Heimer, T., S. T. D’Arcangelis, F. Farzad, J. M. Stipkala, y G. J. Meyer, An Acetylacetonate-Based Semiconductor-Sensitizer Linkage, Inorganic Chemistry, 35, 5319-5324 (1996).

Kalyanasundaram, K., Photochemistry of Polypyridine and Porphyrin Complexes, Academic Press, Boca Raton (1992).

Kelly, C., F. Farzad, D. W. Thompson, J. M. Stipkala y G. J. Meyer, Cation-Controlled Interfacial Charge Injection in Sensitized Nanocrystalline $\mathrm{TiO}_{2}$, Langmuir, 15, 7047-7054 (1999).

Kelly, C., F. Farzad, D. W. Thompson, y G. J. Meyer, Excited-State Deactivation of Ruthenium(II) Polypyridyl Chromophores Bound to Nanocrystalline TiO2 Mesoporous Thin Films, Langmuir, 15, 731-737 (1999).

McCauley, K. M., S. R. Wilson y W. van der Donk, Synthesis and Characterization of Chlorinated Alkenylcobaloximes to Probe the Mechanism of Vitamin $B_{12}$-Catalyzed Dechlorination of Priority Pollutants, Inorganic Chemistry, 41, 393-404 (2002).

Meyer, T. J., Excited-State Electron Transfer, Progress in Inorganic Chemistry 30, 389-439 (1982).

Probst, B., M. Guttentag, A. Rodenberg, P. Hamm y R. Alberto, Photocatalytic $\mathrm{H}_{2}$ Production from Water with Rhenium and Cobalt Complexes, Inorganic Chemistry, 50, 3404-12 (2011).

Schrauzer, G. N., Organocobalt chemistry of vitamin $B_{12}$ model compounds (cobaloximes), Accounts of Chemical Research, 1, 97-103 (1968).

Wen, F., J. Yang, X. Zong, B. Ma, D. Wang, C. Li, Photocatalytic $\mathrm{H}_{2}$ production on hybrid catalyst system composed of inorganic semiconductor and cobaloximes catalysts, Journal of Catalysis, 281, 318-324 (2011).

Zhang, P., M. Wang, J. Dong, X. Li, F. Wang, L. Wu y L. Sun, Photocatalytic Hydrogen Production from Water by Noble-Metal-Free Molecular Catalyst Systems Containing Rose Bengal and the Cobaloximes of $B F_{x}$-Bridged Oxime Ligands, The Journal Of Physical Chemistry C, 114, 15868-15874 (2010). 
